\title{
Estudos de séries temporais de poluição atmosférica por queimadas e saúde humana
}

\author{
Time series studies of air pollution by fires \\ and the effects on human health
}

Cleber Nascimento do Carmo ${ }^{1}$

Sandra de Souza Hacon ${ }^{2}$

\footnotetext{
${ }^{1}$ Universidade Federal do Rio de Janeiro, Campus Macaé. R. Aluísio da Silva Gomes 50, Granja dos Cavaleiros. 27.930-560 Macaé RJ Brasil. cleber@im.ufri.br ${ }^{2}$ Dept Endemias Samuel Pessoa, Escola Nacional de Saúde Publica, Fiocruz.
}

\begin{abstract}
Burnoffs (intentional fires for agricultural purposes) and forest fires of large proportions have been observed in various regions of the planet. Exposure to high levels of air pollutants emitted by fires can be responsible for various harmful effects on human health. In this article, the literature on estimating acute effects of air pollution on human health by fires in the regions with the highest number of fires on the planet, using a time series approach is summarized. An attempt was made to identify gaps in knowledge. The study consisted of a narrative review, in which the characteristics of the selected studies were grouped by regions of the planet with a higher incidence of burnoffs: Amazon, America, Australia and Asia. The results revealed a large number of studies in Australia, few studies in the Amazon and great heterogeneity in the results on the significant effects on human health.
\end{abstract}

Key words Burnoffs, Human health, Time series
Resumo Queimadas e incêndios florestais de grandes proporções têm sido observados em diversas regiões do planeta. A exposição a altos níveis de poluentes atmosféricos emitidos por queimadas podem causar uma variedade de danos à saúde humana. Neste artigo, sumarizamos a literatura existente sobre estimação de efeitos agudos da poluição atmosférica por queimadas na saúde humana nas regiões em que estas estão concentradas em maior número no planeta, utilizandose abordagem de séries temporais. Também se buscou identificar lacunas de conhecimento. O estudo consistiu de uma revisão narrativa, em que as características dos estudos selecionados foram agrupadas pelas regiões do planeta em que há maior incidência de queimadas: Amazônia, Estados Unidos, Austrália e Ásia. Os resultados apontaram concentração de estudos na Austrália, poucos estudos realizados na Amazônia e grande heterogeneidade nos resultados sobre efeitos significativos na saúde humana.

Palavras-chave Queimadas, Saúde humana, Séries temporais 


\section{Introdução}

As queimadas têm chamado atenção e gerado alarme na sociedade desde o início da década de $1980^{1,2}$. Esta preocupação tem sido particularmente evidente na Amazônia e nas florestas tropicais da Ásia, por serem regiões que concentram a maior parte dos incêndios florestais no planeta. Mas incêndios florestais de grandes proporções têm sido observados em regiões da Austrália, Europa e oeste dos Estados Unidos.

Riscos para a saúde humana, destruição de recursos florestais e de biodiversidade, acidentes de transporte e o impacto negativo em diversos setores da economia devido às queimadas têm sido amplamente divulgados ${ }^{3-6}$.

Nos Estados Unidos, destaque é dado ao Estado da Califórnia, que apresentou uma sequência de incêndios florestais de grandes proporções nas décadas de 1980, 1990 e 2000. Em 2007, particularmente, a região teve o pior episódio de queimadas de sua história recente, com prejuízos estimados em 1 bilhão de dólares, destruição de 1.500 residências, afetando uma população de 1 milhão de pessoas, aproximadamente ${ }^{7,8}$. Em fevereiro de 2009, episódio semelhante ocorreu na Austrália, no que foi considerado o segundo maior incêndio da história do país e ficou conhecido como "sábado negro", com 173 óbitos, 2.000 domicílios destruídos e uma área devastada de mais 350.000 hectares?.

No Brasil, os incêndios florestais têm ocorrido, predominantemente, nas porções leste e sul da bacia Amazônica ${ }^{10,11}$.

O primeiro levantamento nacional sobre incêndios florestais foi feito em $1983^{12}$. Naquele ano foram registrados 227 incêndios florestais, queimando uma área 22.269 hectares. No entanto, devido ao fato de muitas instituições públicas não terem registros daquela época, certamente o número real de focos de queimadas foi superior ao registrado pelo levantamento ${ }^{13}$.

É bem conhecido que a exposição a altos níveis de poluição atmosférica pode causar efeitos adversos à saúde humana, principalmente em crianças e idosos ${ }^{14}$. Diversos episódios de severa poluição atmosférica ocorreram em vários países industrializados durante o século XX, provocando graves efeitos na saúde pública, como o caso de Londres, em $1952^{15}$. Diretrizes de qualidade do ar foram estabelecidas com base em estudos sobre tais episódios de poluição atmosférica e são atualizadas segundo resultados mais recentes ${ }^{16}$. No entanto, a qualidade do ar melhorou significativamente nesses países desde a dé- cada de 1970, mas agravos à saúde humana causados pela exposição aos poluentes atmosféricos continuam sendo uma preocupação pública, motivados por um número elevado de estudos de séries temporais epidemiológicas que mostram associações positivas pequenas, mas estatisticamente significativas entre os poluentes e aumentos na morbimortalidade por causas cardiorrespiratórias ${ }^{17-19}$.

Os efeitos da poluição atmosférica por queimadas na saúde humana ainda são pouco estudados, embora seus níveis diários ultrapassem muitas vezes as medições de grandes centros urbanos poluídos ${ }^{20,21}$. Todavia, os estudos existentes indicam que seus efeitos vão além de procuras imediatas aos hospitais para atendimentos por causas cardiorrespiratórias, podendo perdurar por diversos dias ${ }^{21-23}$.

Pesquisas sobre os efeitos da poluição atmosférica na saúde humana evoluíram a partir de estudos descritivos dos fenômenos iniciais de grandes aumentos nos efeitos adversos à saúde após episódios extremos de poluição do ar até as análises de séries temporais.

Neste artigo, sumarizamos a literatura existente sobre a estimação de efeitos agudos da poluição atmosférica por queimadas na saúde humana nas regiões que concentram o maior número de queimadas no planeta. Foi utilizada abordagem de séries temporais, pois tal metodologia assume papel fundamental nos estudos atuais e na configuração de padrões aceitáveis de qualidade do ar para a saúde humana.

Também se buscou identificar lacunas de conhecimento e questões de pesquisa detalhadas, que são essenciais para o sucesso das análises e divulgação de medidas e políticas preventivas.

\section{Exposição à poluição atmosférica causada por queimadas \\ e seus efeitos na saúde humana}

É conhecido que fumaça de qualquer origem produz efeitos negativos na saúde humana, especialmente em crianças, idosos e pessoas com sistema cardiorrespiratório debilitado ${ }^{24}$. Diversas revisões têm discutido os impactos na saúde humana da poluição por queima de biomassa ${ }^{25-}$ 27. Embora, a ênfase desses trabalhos tenha sido em efeitos da poluição atmosférica por queima de madeira em ambientes internos e sobre o fumo de cigarros, muitas de suas conclusões podem ser extrapoladas para o entendimento dos efeitos das queimadas florestais na saúde humana. Porém, em tais estudos, geralmente, a exposição 
é investigada em concentrações conhecidas e por uma duração de tempo específica ${ }^{28}$.

$\mathrm{Na}$ região Amazônica, os incêndios florestais são sazonais, ocorrendo principalmente no período compreendido entre os meses de junho a outubro, em que baixas umidades e ausência de chuvas predominam. Em relação às queimadas em áreas remotas desta região, os poluentes gasosos e o material particulado apresentam efeitos diretos para o sistema respiratório, em especial para as crianças ${ }^{29}$. As queimadas representam a maior ameaça à integridade biológica da região, elas alteram o balanço de radiação atmosférica, afetando o ecossistema amazônico, além de trazer impactos negativos à economia $\mathrm{e}$ à saúde pública ${ }^{3,29-31}$.

Além de conter grandes quantidades de material particulado, há a presença de hidrocarbonetos aromáticos policíclicos (PAH), monóxido de carbono, aldeídos, ácidos inorgânicos que se alteram, algumas vezes, rapidamente, com o tempo, temperatura, luz solar, interações com outros poluentes, vapor d'água e superfícies ${ }^{21,32,33}$.

O material particulado (PM) é o poluente que apresenta maior toxicidade e que tem sido o mais estudado. O PM é, geralmente, classificado pelo seu tamanho, uma vez que esse parâmetro determina a deposição dentro do sistema respiratório ${ }^{34}$. Partículas menores penetram mais profundamente nos pulmões. A maior parte do PM emitido em queima de biomassa possui diâmetro inferior a $0,1 \mu \mathrm{m}$ ou se situa entre $0,1 \mu \mathrm{m}$ e 2,5 $\mu \mathrm{m}^{35}$. Entretanto, a legislação para padrões de qualidade do ar foi, originalmente, formulada para quantificar PM com diâmetro igual ou inferior a $10 \mu \mathrm{m}^{36}$. Nos Estados Unidos, a média anual de $\mathrm{PM}_{10}$ considerada segura para a saúde humana é de até $50 \mu \mathrm{mm}^{-3}$, com uma exposição máxima por um período de $24 \mathrm{~h}$ estimada em $150 \mu \mathrm{mm}^{-37}$. A regulamentação sobre os níveis aceitáveis de $\mathrm{PM}_{2,5}$ é relativamente nova (julho de 1997), com uma média anual estabelecida em até $15 \mu^{-3}$ e exposição máxima por período de $24 \mathrm{~h}$ estimada em até $35 \mu \mathrm{mm}^{-3} 38$.

No Brasil, os padrões de qualidade do ar adotados são estabelecidos pela legislação do Conselho Nacional de Meio Ambiente - Conama no $03 / 1990^{39}$. A legislação brasileira estabelece limites aceitáveis apenas para o $\mathrm{PM}_{10}$ que é de 60 $\mu \mathrm{mm}^{-3}$ para a média diária. Embora de fundamental importância para a avaliação de impactos da poluição atmosférica por queimadas na saúde humana, poucos estudos têm investigado diretamente os efeitos do $\mathrm{PM}_{2,5}$ na saúde humana, principalmente, na Amazônia ${ }^{29,40,41}$. Mas, sim- plesmente, porque estas partículas não são tão facilmente mensuráveis quanto o $\mathrm{PM}_{10}{ }^{42}$.

\section{Monitoramento das queimadas e fontes de dados}

Diversos países possuem redes de estações de monitoramento de qualidade do ar como meio de mensurar os níveis de poluição atmosférica. Mesmo sendo de fundamental importância, na Indonésia não há um sistema integrado capaz de fornecer em tempo real informação sobre a qualidade do ar, cada governo a conduz de maneira independente de cada uma das capitais das 27 províncias $^{43}$. Um sistema de monitoramento mais sofisticado está disponível na Malásia e em Singapura. Na Singapura é adotado um índice padrão de poluição (PSI) utilizado pela Agência de Proteção Ambiental Americana (USEPA). Na Malásia, por sua vez, adota-se um índice de poluição do ar (API) com metodologia similar ${ }^{44}$. Durante episódios de queimadas, tais índices são, majoritariamente, baseados nas concentrações de material particulado, tendo em vista que o PM ultrapassa em muito as demais concentrações de poluentes $^{44}$.

Informação sobre saúde pública relacionada com incêndios florestais na Austrália está disponível para o público em geral através do Ministério da Saúde daquele país, particularmente, sobre aqueles Estados que possuem histórico de episódios de queimadas. Além disso, os australianos dispõem de um modelo numérico de previsão de poluentes atmosféricos (Australian Air Quality Forecasting System, AAQFS) para as cidades de Melbourne, Sydney e arredores ${ }^{45}$.

No Brasil, desde a década de 80, o Instituto Nacional de Pesquisas Espaciais (INPE) vem sofisticando seu sistema de detecção de queimadas, que a partir de 1998 dá ênfase particular à Amazônia. Os focos de queimadas passaram as ser obtidos por imagens dos satélites NOAA (National Oceanic and Atmospheric Administration) quatro vezes ao dia e mais recentemente também nas imagens do GOES-Leste (Geostationary Operational Environmental Satellite) oito ou mais vezes ao dia, e TERRA e AQUA duas vezes por dia cada, sendo em seguida integrados a dois sistemas de informações geográficas acessíveis na Internet ${ }^{30,46}$.

Entre os muitos produtos deste sistema, destacam-se os mapas de risco de queima da vegetação observado, previsto e climático, banco de dados de queimadas desde 1992; a expansão do monitoramento das queimadas para vários paí- 
ses vizinhos ao Brasil, entre outros. Todas estas informações estão disponíveis ao público em geral através do acesso a um banco de dados online onde coordenadas dos focos de queimadas podem ser obtidas ${ }^{47}$.

Devido ao elevado custo operacional e às dificuldades de infraestrutura, a implantação de uma rede automática de monitoramento de poluentes atmosféricos na região amazônica seria impraticável. Neste sentido, pesquisadores do INPE desenvolveram o modelo numérico atmosférico CCATT-BRAMS (Coupled Chemistry Aerosol and Tracers Transport Model to the Brazilian Regional Atmospheric Modeling System) que estima a concentração de poluentes atmosféricos derivados da queima de biomassa ${ }^{48}$. As principais vantagens deste procedimento estão em permitir a obtenção do prognóstico simultâneo do estado atmosférico dos pontos de vista meteorológico e de qualidade do ar, assim como o estudo da interação entre o contaminante e a própria atmosfera ${ }^{30}$.

Parcialmente devido ao problema dos grandes episódios de queimadas, houve um aumento na quantidade de estações de monitoramento na última década. Além disso, observa-se uma especial atenção para a mensuração de particulados, tendo em vista que as queimadas emitem estes contaminantes em quantidade muito superior aos demais poluentes ${ }^{43}$.

\section{Pesquisa atual com séries temporais}

A pesquisa sobre efeitos da poluição atmosférica na saúde humana acumulou muita informação oriunda de diversos ramos do conhecimento, que incluem a toxicologia, a análise de risco, a epidemiologia e a bioestatística ${ }^{21,49,50}$.

Para estimar os efeitos de curto prazo na saúde humana da poluição atmosférica - seja esta urbana ou oriunda de queimadas - a abordagem mais utilizada nas últimas duas décadas tem sido um estudo epidemiológico ecológico de séries temporais ${ }^{18}$.

Os resultados de estudos de séries temporais são importantes para as agências reguladoras ambientais, que determinam os padrões de qualidade do ar considerados seguros para a saúde humana ${ }^{51}$. Além disso, desempenham um papel crucial na discussão recente sobre padrões apropriados de qualidade do ar para PM nos Estados Unidos e em diversos países ${ }^{51,52}$. Em tal desenho de estudo, os dados utilizados são agregados tanto para a exposição quanto para os desfechos de saúde. As inferências sobre a associação entre a exposição e o desfecho consistem em relacionar as flutuações de curto prazo na série de agravos à saúde com aquelas na variável de exposição ${ }^{48}$. A importância de se remover efeitos de longa duração como tendência e sazonalidade, condições meteorológicas e autocorrelação serial em tais estudos é amplamente conhecida para garantir que as estimativas de efeitos dos poluentes atmosféricos não estejam confundidas por nenhuma outra variável ${ }^{53,54}$.

Nas análises de séries temporais, modelos de regressão são utilizados para estimar o aumento no risco relativo para um desfecho de saúde, como mortalidade ou morbidade por doenças respiratórias, associados a um aumento unitário nos níveis de determinado poluente $e^{49,55}$.

Modelos aditivos generalizados (GAM) têm se tornado uma ferramenta padrão de análise de séries temporais em estudos de poluição atmosférica e saúde ${ }^{49}$. Tais modelos possuem a importante característica de permitir a modelagem de relações não lineares que não apresentam uma forma definida. Para quantificar os efeitos da poluição atmosférica na saúde humana, assume-se que o poluente está linearmente relacionado com os indicadores de saúde. Entretanto, as relações entre estes e as potenciais variáveis de confundimento não podem ser facilmente parametrizadas e funções suavizadoras são necessárias para a modelagem destes termos.

Desde a penúltima década, o seguinte modelo se tornou padrão em estudos de poluição atmosférica e saúde humana, seja em áreas urbanas ou poluição atmosférica por queimadas:

$$
\begin{aligned}
& Y_{t} \sim \operatorname{Poisson}(\mu) \\
& g[E(Y \backslash X, T)]=g(\mu)=\eta=X \beta+\Sigma f_{i}\left(T_{i}\right)
\end{aligned}
$$

Neste modelo, $Y$ representa o desfecho de saúde sob estudo. Por se tratar de dados de contagem, um dos modelos de probabilidade mais adequados é o de Poisson. O vetor $X$ representa o poluente e as variáveis indicadoras; $T_{i}$ representa o tempo transcorrido do início da série até o dia corrente, variáveis meteorológicas e demais pontenciais covariáveis; $g$ é a função de ligação. O interesse maior está em estimar $\beta$, o vetor que descreve a variação percentual no logaritmo da média do desfecho de saúde considerado para a variação em uma unidade na exposição. A curva suavizada, por meio dos $f_{i}\left(T_{i}\right)$ permite descrever e, até mesmo, revelar possíveis não linearidades nas relações estudadas, uma vez que não apresenta a estrutura rígida de uma função paramétrica.

Outro modelo comumente utilizado nos estudos de poluição atmosférica e saúde é o modelo linear generalizado (GLM) com splines para- 
métricas. O GLM utiliza o método de máxima verossimilhança para estimar o vetor de parâmetros $\beta$ e os cálculos são feitos por meio de um procedimento iterativo ${ }^{56}$. Diversas análises sugerem que a diferença entre as estimativas de risco à saúde pelos modelos GAM e GLM depende da forma das funções não lineares de tempo, climáticas e de sazonalidade, do grau de ajustamento para os fatores de confundimento e a correlação entre os preditores que variam no tempo ${ }^{50,57}$.

A seguir, primeiramente, apresenta-se um panorama sobre os estudos feitos na Amazônia (Brasil) e na Califórnia (Estados Unidos), seguido por outros realizados na Ásia e na Austrália. Foram consideradas apenas pesquisas publicadas nos idiomas inglês e português, em periódicos internacionais, para estimar as associações entre a exposição à poluição por queimadas e os agravos cardiorrespiratórios, nas últimas duas décadas. Não pretendemos fazer uma revisão sistemática formal ou metanálise dos trabalhos publicados neste campo do conhecimento, mas discutir questões consideradas de vital importância, com base na literatura recente e nas informações necessárias, para auxiliar os tomadores de decisão em ações preventivas. Nos Quadros 1, 2, 3 e 4 são apresentados alguns detalhes sobre os estudos que mensuraram concentrações de PM e a relacionaram com desfechos de saúde.

\section{Amazônia}

$\mathrm{Na}$ Amazônia, estudos sobre impactos da poluição atmosférica por queimadas na saúde humana ainda são relativamente recentes. Estudos de monitoramento de aerossóis vêm sendo desenvolvidos desde 1992 na região pelo Experimento de Grande Escala da Biosfera e Atmosfera da Amazônia (LBA), do Grupo de Estudos de Poluição do Ar (GEPA), pertencente ao Instituto de Física da Universidade de São Paulo (IF-USP).

Foram encontrados três estudos epidemiológicos com desenho ecológico de séries temporais na região amazônica. Os estudos foram realizados nos Estados brasileiros do Acre $^{41}$ e do Mato Grosso ${ }^{29,40}$.

No ano de 2005, a Amazônia foi assolada por um prolongado e intenso período de seca e por inúmeros focos de incêndios florestais. Estimase que a população atingida pela fumaça das

Quadro 1. Estudos que investigaram a associação entre poluição atmosférica por queimadas florestais e desfechos cardiorrespiratórios na Amazônia.

\begin{tabular}{|c|c|c|c|c|c|c|}
\hline \multicolumn{7}{|c|}{ Amazônia } \\
\hline Estudo & $\begin{array}{l}\text { Período de } \\
\text { estudo }\end{array}$ & $\begin{array}{l}\text { Área de } \\
\text { Estudo }\end{array}$ & $\begin{array}{l}\text { Variável de } \\
\text { Exposição }\end{array}$ & $\begin{array}{l}\text { Desfecho de saúde e } \\
\text { população de estudo }\end{array}$ & $\begin{array}{c}\text { Metodologia } \\
\text { analítica }\end{array}$ & $\begin{array}{l}\text { Resultados } \\
\text { significativos }\end{array}$ \\
\hline $\begin{array}{c}\text { Mascarenhas } \\
\text { et al. }{ }^{41}\end{array}$ & $\begin{array}{l}\text { Setembro } \\
\text { de } 2005\end{array}$ & $\begin{array}{l}\text { Rio } \\
\text { Branco, } \\
\text { AC }\end{array}$ & $\mathrm{PM}_{2,5}$ & $\begin{array}{c}\text { Atendimento } \\
\text { ambulatorial por } \\
\text { doenças respiratórias } \\
\text { em todas as faixas } \\
\text { etárias }\end{array}$ & $\begin{array}{l}\text { Análise de } \\
\text { regressão } \\
\text { simples das } 2 \\
\text { séries } \\
\text { temporais }\end{array}$ & $\begin{array}{l}\text { Associação significativa, } \\
\text { mas sem dados sobre riscos } \\
\text { relativos. }\end{array}$ \\
\hline $\begin{array}{c}\text { Carmo et } \\
\text { al. }^{29}\end{array}$ & $\begin{array}{l}\text { Janeiro de } \\
2004 \text { a } \\
\text { dezembro } \\
\text { de } 2005\end{array}$ & $\begin{array}{l}\text { Alta } \\
\text { Floresta, } \\
\text { MT }\end{array}$ & $\mathrm{PM}_{2,5}$ & $\begin{array}{c}\text { Atendimento } \\
\text { Ambulatorial por } \\
\text { doenças respiratórias } \\
\text { de crianças e de } \\
\text { idosos }\end{array}$ & $\begin{array}{l}\text { Regressão de } \\
\text { Poisson } \\
\text { (GAM) }\end{array}$ & $\begin{array}{l}\text { Aumentos de } 10 \mu \mathrm{g} / \mathrm{m}^{3} \text { nos } \\
\text { níveis de } \mathrm{PM}_{2,5} \text { associados a } \\
\text { aumentos de } 2,9 \text { e } 2,6 \% \\
\text { nos atendimentos por } \\
\text { doenças respiratórias de } \\
\text { crianças no } 6^{\circ} \text { e } 70 \text { dias } \\
\text { subsequentes à exposição. }\end{array}$ \\
\hline $\begin{array}{l}\text { Ignotti et } \\
\text { al. }^{40}\end{array}$ & $\begin{array}{l}\text { Janeiro a } \\
\text { dezembro } \\
\text { de } 2005\end{array}$ & $\begin{array}{c}\text { Alta } \\
\text { Floresta e } \\
\text { Tangará da } \\
\text { Serra, MT }\end{array}$ & $\mathrm{PM}_{2,5}$ & $\begin{array}{c}\text { Internações por } \\
\text { doenças respiratórias } \\
\text { de crianças e de } \\
\text { idosos }\end{array}$ & $\begin{array}{l}\text { Regressão de } \\
\text { Poisson } \\
\text { (GAM) }\end{array}$ & $\begin{array}{l}\text { Aumentos de } 10 \mu \mathrm{g} / \mathrm{m}^{3} \text { nos } \\
\text { níveis de } \mathrm{PM}_{2,5} \text { associados a } \\
\text { aumentos de até } 6 \% \text { nas } \\
\text { internações de crianças e } \\
\text { de } 6,8 \% \text { dos idosos. } \\
\text { Nenhuma associação foi } \\
\text { encontrada em Tangará da } \\
\text { Serra. }\end{array}$ \\
\hline
\end{tabular}


Quadro 2. Estudos que investigaram a associação entre poluição atmosférica por queimadas florestais e desfechos cardiorrespiratórios na Califórnia.

\begin{tabular}{|c|c|c|c|c|c|c|}
\hline \multicolumn{7}{|c|}{ Califórnia } \\
\hline Estudo & $\begin{array}{l}\text { Período de } \\
\text { estudo }\end{array}$ & $\begin{array}{l}\text { Área de } \\
\text { Estudo }\end{array}$ & $\begin{array}{l}\text { Variável de } \\
\text { Exposição }\end{array}$ & $\begin{array}{l}\text { Desfecho de saúde e } \\
\text { população de estudo }\end{array}$ & $\begin{array}{c}\text { Metodologia } \\
\text { analítica }\end{array}$ & $\begin{array}{l}\text { Resultados } \\
\text { significativos }\end{array}$ \\
\hline Jacobs et al.$^{67}$ & $\begin{array}{l}\text { Janeiro de } \\
1983 \text { a } \\
\text { dezembro } \\
\text { de } 1992\end{array}$ & $\begin{array}{l}\text { Butte } \\
\text { County, } \\
\text { CA }\end{array}$ & \begin{tabular}{|}
$\mathrm{PM}_{10}, \mathrm{O}_{3}, \mathrm{CO}$, \\
$\mathrm{COH}$ e Total de \\
área queimada
\end{tabular} & $\begin{array}{c}\text { Internações } \\
\text { hospitalares por asma } \\
\text { em todas as faixas } \\
\text { etárias }\end{array}$ & $\begin{array}{c}\text { Regressão de } \\
\text { Poisson } \\
\text { (GLM) }\end{array}$ & $\begin{array}{c}\text { Aumentos de } 1 \text { acre no total } \\
\text { de área queimada associados } \\
\text { a aumentos de } 23 \% \text { nas } \\
\text { internações por asma. }\end{array}$ \\
\hline $\begin{array}{c}\text { Viswanathan } \\
\text { et al. }{ }^{68}\end{array}$ & $\begin{array}{l}\text { Setembro } \\
\text { de } 2003 \text { a } \\
\text { novembro } \\
\text { de } 2003\end{array}$ & $\begin{array}{c}\text { San Diego, } \\
\text { CA }\end{array}$ & $\begin{array}{c}\mathrm{PM}_{10}, \mathrm{PM}_{2,5} \\
\mathrm{CO}, \mathrm{O}_{3}, \mathrm{NO}_{2} \mathrm{e} \\
\mathrm{SO}_{2}\end{array}$ & $\begin{array}{c}\text { Atendimento } \\
\text { ambulatorial por } \\
\text { doenças respiratórias } \\
\text { em todas as faixas } \\
\text { etárias }\end{array}$ & $\begin{array}{c}\text { Análise } \\
\text { descritiva de } \\
\text { séries } \\
\text { temporais, } \\
\text { análise de } \\
\text { correlação }\end{array}$ & $\begin{array}{l}\text { Associação significativa entre } \\
\text { PM e os atendimentos } \\
\text { ambulatoriais, mas sem } \\
\text { dados sobre riscos relativos. }\end{array}$ \\
\hline
\end{tabular}

Quadro 3. Estudos que investigaram a associação entre poluição atmosférica por queimadas florestais e desfechos cardiorrespiratórios na Ásia.

\begin{tabular}{|c|c|c|c|c|c|c|}
\hline \multicolumn{7}{|c|}{ Ásia } \\
\hline Estudo & $\begin{array}{l}\text { Período de } \\
\text { estudo }\end{array}$ & $\begin{array}{l}\text { Área de } \\
\text { Estudo }\end{array}$ & $\begin{array}{l}\text { Variável de } \\
\text { Exposição }\end{array}$ & $\begin{array}{l}\text { Desfecho de saúde e } \\
\text { população de estudo }\end{array}$ & $\begin{array}{c}\text { Metodologia } \\
\text { analítica }\end{array}$ & $\begin{array}{l}\text { Resultados } \\
\text { significativos }\end{array}$ \\
\hline Chew et al. ${ }^{72}$ & $\begin{array}{l}\text { Janeiro de } \\
1990 \text { a } \\
\text { dezembro } \\
\text { de } 1994\end{array}$ & Singapura & $\begin{array}{c}\mathrm{PM}_{10}, \mathrm{SO}_{2} \\
\mathrm{NO}_{2}, \mathrm{O}_{3}\end{array}$ & $\begin{array}{c}\text { Internações } \\
\text { hospitalares e } \\
\text { Atendimento } \\
\text { ambulatorial por } \\
\text { asma em crianças e } \\
\text { adolescentes }\end{array}$ & $\begin{array}{l}\text { Regressão } \\
\text { linear } \\
\text { múltipla } \\
\text { (GLM) }\end{array}$ & $\begin{array}{l}\text { Aumentos de } \mathrm{SO}_{2} \text { e PM10 } \\
\text { associados com aumentos de } \\
2.9 \% \text { e } 5.8 \% \text { nos } \\
\text { atendimentos por doenças } \\
\text { respiratórias. }\end{array}$ \\
\hline $\begin{array}{l}\text { Emmanuel } \\
\text { et al. }{ }^{5}\end{array}$ & $\begin{array}{c}\text { Agosto a } \\
\text { novembro } \\
\text { de } 1997\end{array}$ & Singapura & $\mathrm{PM}_{10}$ & $\begin{array}{l}\text { Atendimento } \\
\text { Ambulatorial, } \\
\text { internações e } \\
\text { mortalidade em todas } \\
\text { as faixas etárias }\end{array}$ & $\begin{array}{c}\text { Análise } \\
\text { descritiva de } \\
\text { séries } \\
\text { temporais }\end{array}$ & $\begin{array}{l}\text { Aumentos de PM de } 50 \mu \mathrm{g} / \\
\mathrm{m}^{3} \text { a } 150 \mu \mathrm{g} / \mathrm{m}^{3} \text { associados } \\
\text { com aumentos de } 12 \% \text { nos } \\
\text { atendimentos por doenças } \\
\text { do trato respiratório } \\
\text { superior, } 37 \% \text { por asma, } \\
26 \% \text { por rinite. Não foram } \\
\text { encontradas associações para } \\
\text { internações e para } \\
\text { mortalidade. }\end{array}$ \\
\hline Sastry et al. ${ }^{71}$ & $\begin{array}{l}\text { Janeiro de } \\
1996 \text { a } \\
\text { dezembro } \\
\text { de } 1997\end{array}$ & Malásia & $\mathrm{PM}_{10}$ & $\begin{array}{c}\text { Mortalidade por } \\
\text { todas as causas e por } \\
\text { doenças respiratórias, } \\
\text { cardiovasculares }\end{array}$ & $\begin{array}{l}\text { Regressão de } \\
\text { Poisson } \\
\text { (GLM) }\end{array}$ & $\begin{array}{l}\text { Aumento de PM associado } \\
\text { com aumento de } \\
\text { mortalidade por todas as } \\
\text { causas para idosos, mas sem } \\
\text { evidências para mortaldiade } \\
\text { por causas respiratórias. }\end{array}$ \\
\hline Mott et al. ${ }^{70}$ & $\begin{array}{l}\text { Janeiro de } \\
1995 \text { a } \\
\text { dezembro } \\
\text { de } 1998\end{array}$ & Malásia & $\begin{array}{l}\text { Air pollution } \\
\text { Index (API) }\end{array}$ & $\begin{array}{l}\text { Internações por } \\
\text { asma, causas } \\
\text { respiratórias, } \\
\text { cardiovasculares }\end{array}$ & $\begin{array}{l}\text { Modelos } \\
\text { Holt- } \\
\text { Winters }\end{array}$ & $\begin{array}{l}\text { Aumento das internações } \\
\text { por asma e COPD durante } \\
\text { as queimadas; maior } \\
\text { probabilidade de } \\
\text { rehospitalização de idosos } \\
\text { durante as queimadas. }\end{array}$ \\
\hline
\end{tabular}


Quadro 4. Estudos que investigaram a associação entre poluição atmosférica por queimadas florestais e desfechos cardiorrespiratórios na Austrália.

\begin{tabular}{|c|c|c|c|c|c|c|}
\hline \multicolumn{7}{|c|}{ Austrália } \\
\hline Estudo & $\begin{array}{l}\text { Período de } \\
\text { estudo }\end{array}$ & $\begin{array}{l}\text { Área de } \\
\text { Estudo }\end{array}$ & $\begin{array}{l}\text { Variável de } \\
\text { Exposição }\end{array}$ & $\begin{array}{l}\text { Desfecho de saúde e } \\
\text { população de estudo }\end{array}$ & $\begin{array}{c}\text { Metodologia } \\
\text { analítica }\end{array}$ & $\begin{array}{l}\text { Resultados } \\
\text { significativos }\end{array}$ \\
\hline Smith et al. ${ }^{79}$ & $\begin{array}{c}\text { Dezembro } \\
\text { de } 1992 \text { a } \\
\text { janeiro de } \\
1993 \text { e } \\
\text { dezembro } \\
\text { de } 1993 \text { a } \\
\text { janeiro de } \\
1994\end{array}$ & $\begin{array}{l}\text { Western } \\
\text { Sydney }\end{array}$ & $\mathrm{PM}_{10}$ & $\begin{array}{l}\text { Atendimentos de } \\
\text { emergência por asma, } \\
\text { todas as faixas etárias. }\end{array}$ & $\begin{array}{l}\text { Regressão de } \\
\text { Poisson } \\
\text { (GLM) }\end{array}$ & $\begin{array}{l}\text { Nenhum aumento } \\
\text { significativo dos } \\
\text { atendimentos por asma } \\
\text { durante os episódios de } \\
\text { incêndios florestais. }\end{array}$ \\
\hline $\begin{array}{c}\text { Jalaludin et } \\
\text { al. }^{77}\end{array}$ & $\begin{array}{c}\text { Janeiro de } \\
1994\end{array}$ & Sydney & $\mathrm{PM}_{10}$ & $\begin{array}{c}\text { Crianças com } \\
\text { histórico de sibilância } \\
\text { nos últimos } 12 \text { meses } \\
\text { de } 6 \text { escolas } \\
\text { primárias. }\end{array}$ & $\begin{array}{c}\text { Modelos de } \\
\text { Equação de } \\
\text { Estimação } \\
\text { Generalizadas } \\
\text { (GEE) }\end{array}$ & $\begin{array}{l}\text { Nenhuma redução } \\
\text { significativa de função } \\
\text { pulmonar das crianças } \\
\text { durante as queimadas. }\end{array}$ \\
\hline $\begin{array}{c}\text { Johnston et } \\
\text { al. }^{80}\end{array}$ & $\begin{array}{c}\text { Abril a } \\
\text { dezembro } \\
\text { de } 2000\end{array}$ & Darwin & $\mathrm{PM}_{10}$ & $\begin{array}{c}\text { Internações } \\
\text { hospitalares por } \\
\text { asma, todas as faixas } \\
\text { etárias }\end{array}$ & $\begin{array}{l}\text { Regressão } \\
\text { Binomial } \\
\text { Negativa } \\
\text { (GLM) }\end{array}$ & $\begin{array}{c}\text { Aumentos de PM de } 10 \mu \mathrm{g} / \\
\mathrm{m}^{3} \text { associado com aumentos } \\
\text { de } 20 \% \text { e } 39 \% \text { nas } \\
\text { internações por asma. }\end{array}$ \\
\hline Chen et al. ${ }^{81}$ & $\begin{array}{l}\text { Julho de } \\
1997 \text { a } \\
\text { dezembro } \\
\text { de } 2000\end{array}$ & Brisbane & $\mathrm{PM}_{10}$ & $\begin{array}{c}\text { Internações } \\
\text { hospitalares por } \\
\text { doenças respiratórias, } \\
\text { todas as faixas etárias }\end{array}$ & $\begin{array}{l}\text { Regressão } \\
\text { Binomial } \\
\text { Negativa } \\
\text { (GLM) }\end{array}$ & $\begin{array}{c}\text { Foram encontradas } \\
\text { associações para períodos de } \\
\text { incêndios }(\mathrm{RR}=1.19) \text { e para } \\
\text { períodos sem incêndios } \\
(\mathrm{RR}=1.13)\end{array}$ \\
\hline $\begin{array}{c}\text { Hanigan et } \\
\text { al. }^{76}\end{array}$ & $\begin{array}{c}\text { Abril a } \\
\text { novembro, } \\
\text { de } 1996 \text { a } \\
2005\end{array}$ & Darwin & $\mathrm{PM}_{10}$ & $\begin{array}{c}\text { Internações } \\
\text { hospitalares por } \\
\text { doenças } \\
\text { cardiorrespiratórias, } \\
\text { todas as faixas etárias }\end{array}$ & $\begin{array}{l}\text { Regressão de } \\
\text { Poisson } \\
\text { (GLM) }\end{array}$ & $\begin{array}{c}\text { Aumentos de PM de } 10 \mu \mathrm{g} / \\
\mathrm{m}^{3} \text { associado com aumentos } \\
\text { de } 4.8 \% \text { nas internações por } \\
\text { causas respiratórias. Não } \\
\text { houve associação para } \\
\text { doenças cardiovasculares. }\end{array}$ \\
\hline
\end{tabular}

queimadas tenha sido superior a 400.000 pessoas, com uma área total de florestas devastadas de mais de 300.000 hectares e cerca de U\$ $50 \mathrm{mi}$ lhões de prejuízos financeiros diretos ${ }^{58}$.

Neste contexto, Mascarenhas et al. ${ }^{41}$, com base em dados de atendimento ambulatorial por doenças respiratórias a pacientes de todas as idades no mês de setembro de 2005 na cidade de Rio Branco, capital do Estado do Acre, Brasil, investigaram a associação entre a demanda por atendimentos ambulatoriais e níveis diários de $\mathrm{PM}_{2,5}$, utilizando um estudo ecológico de séries temporais por meio de uma regressão linear simples entre desfecho e exposição. Como resultado, os autores encontraram uma relação positiva entre o material particulado fino e o número de atendimentos por asma.

Os sinais e os sintomas clínicos mais frequentes dos 2.922 pacientes atendidos naquela ocasião foram: tosse $(79 \%)$, febre $(51 \%)$, dispneia $(39 \%)$, dor no peito $(15 \%)$, sibilância $(8 \%)$ e irritação na garganta (4\%). Embora a metodologia adotada por esses autores não tenha sido adequada para a análise e não tenha considerado a possível influência de diversos confundidores, como umidade relativa e temperatura, este estudo tornou-se pioneiro ao tentar investigar os efeitos da poluição atmosférica por queimadas com base num estudo ecológico de séries temporais na região amazônica. 
No ano de 2007, Ignotti et al. ${ }^{59}$ realizaram um estudo descritivo de indicadores de morbimortaliade hospitalar por doenças respiratórias em crianças menores de 5 anos de idade em municípios do Estado do Mato Grosso, líder no ranking de incêndios florestais naquele período. Como resultados, os autores apontaram os municípios de Alta Floresta e Tangará da Serra, como áreas de maior atenção, considerando a exposição aos poluentes atmosféricos liberados nas queimadas.

Tal trabalho motivou a realização de dois estudos que analisaram os efeitos das queimadas na saúde humana naquela região. Carmo et al. ${ }^{29}$ estimaram os efeitos de curto prazo da exposição ao material particulado fino de queimadas na saúde. Os autores utilizaram dados de atendimento ambulatorial por doenças respiratórias de crianças e idosos do município de Alta Floresta, Mato Grosso, Brasil, no período de 2004 a 2005. Com base num modelo aditivo generalizado com regressão de Poisson, foram encontrados resultados significativos para aumentos de $2,6 \%$ e $2,9 \%$ nos atendimentos ambulatoriais por doenças respiratórias de crianças no $6^{\circ}$ e $7^{\circ}$ dias subsequentes à exposição. Não foram encontradas associações significativas nos atendimentos de idosos. Neste estudo, foram utilizados fatores de confundimento comuns em séries temporais epidemiológicas de efeitos da poluição atmosférica: umidade relativa, precipitação, temperatura e variáveis de calendário.

No mesmo ano, Ignotti et al. ${ }^{40}$ realizaram estudo semelhante, porém para os dois municípios do Estado de Mato Grosso com piores indicadores de morbirmortalidade respiratória: Alta Floresta e Tangará da Serra. Neste trabalho, os autores investigaram a associação entre a demanda diária de internações hospitalares por doenças respiratórias de crianças e idosos no ano de 2005. Como fatores de confundimento, foram utilizados: umidade relativa, temperatura e variáveis de calendário. Somente foram encontradas associações significativas para o município de Alta Floresta. Associações significativas foram encontradas para exposições 3, 4, 5 e 6 dias anteriores à admissão hospitalar de crianças e de idosos.

\section{Estados Unidos}

Acima da metade dos incêndios florestais mais severos dos Estados Unidos nos últimos 170 anos ocorreu no Estado da Califórnia ${ }^{60} . \mathrm{Na}-$ cionalmente, as maiores perdas de propriedades e de saúde pública relacionadas a incêndios florestais ocorrem naquele Estado. Mas, ao mesmo tempo, a expansão mobiliária nesta região continua num ritmo acelerado ${ }^{61}$.

Os incêndios florestais nesta região ocorrem, geralmente, durante os ventos de Santa Ana ${ }^{60,61}$. Estes ventos são caracterizados por serem severos, secos, quentes e sopram do Deserto de Mojave para o sul da Califórnia no período compreendido entre os meses de outubro a março ${ }^{62}$. As queimadas na região oeste dos EUA ocorrem, geralmente, devido ao aumento de temperaturas e à redução de precipitação na primavera e verão ${ }^{63}$.

Diversos estudos nos EUA têm avaliado os impactos na saúde humana associados às queimadas. Porém, a maioria faz um estudo comparativo entre a demanda por serviços médicos durante o período de episódios de incêndios florestais com os de controle, em que não há queimadas, por meio de razões de visitas observadas e esperadas ao posto médico e entrevistas ${ }^{64-66}$.

Um estudo de séries temporais realizado na região de Butte, Califórnia, durante 10 anos, sugeriu que as queimadas de resíduos de plantações de arroz estão associadas com internações hospitalares por asma ${ }^{67}$. Como resultado, foi evidenciado que o total de área queimada estava significativamente associado com um aumento do risco de hospitalização por asma e mostrou uma tendência de exposição-resposta. O maior risco de hospitalização foi observado nos dias em que 500 ou mais hectares foram queimados ( risco relativo $=1,23$, IC95\% $=1,09,1,39)$.

Em San Diego, análises descritivas de séries temporais dos dados de vigilância médica mostraram aumentos significativos nos atendimentos de emergência por asma e por problemas respiratórios diversos durante os incêndios florestais de outubro de 2003 na região ${ }^{68}$. Para investigar os impactos dos poluentes gerados pelos incêndios, os autores utilizaram dados de quatro semanas anteriores ao início das queimadas (25 de outubro de 2003), um período de 10 dias durante os episódios de queimadas, e mais quatro semanas após as queimadas. Foram analisados níveis diários de $\mathrm{PM}_{2,5}, \mathrm{PM}_{10}, \mathrm{O}_{3}, \mathrm{CO}$ e $\mathrm{NO}_{2}$.

\section{Ásia}

A prática de limpeza de áreas de florestas por meio do uso de fogo tem resultado em diversos episódios de grandes queimadas de longa duração no Sudeste da Ásia. Os mais severos incêndios florestais da história recente desta região ocorreram nos anos de 1997/1998, paralelamente com o fenômeno climático El Niño, que resultaram na destruição de 11,7 milhões de hectares de floresta, 
propriedades e demais tipos de vegetação ${ }^{2,69}$. Os impactos na saúde humana associados com estes grandes eventos de queimadas e por outros de menores proporções têm sido documentados na literatura por diversos autores ${ }^{5,70,71}$.

Uma análise dos atendimentos de emergência e hospitalizações por asma em crianças em Singapura durante o período de 1990 a 1994 encontrou associações significativas com níveis di-

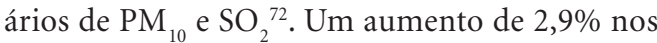
atendimentos de emergência por asma esteve associado com crescimento de $20 \mu \mathrm{g} / \mathrm{m}^{3}$ nos níveis diários de $\mathrm{SO}_{2}$, um dia após a exposição. Com o $\mathrm{PM}_{10}$, para cada incremento de $20 \mu \mathrm{g} / \mathrm{m}^{3}$ em seus níveis diários, aumentos de 5,8\% nos atendimentos de emergência foram verificados.

Estudos de vigilância e monitoramento do ar durante os maiores episódios de queimadas do sudeste asiático em 1997-1998 indicaram reflexos na saúde pública. Em Singapura, durante a época dos incêndios, houve um aumento de 30\% na procura por atendimentos médicos por doenças relacionadas à exposição à fumaça ${ }^{21}$. Emmanuel et al. ${ }^{5}$, por meio de análise descritiva de séries temporais, encontraram associações entre desfecho de saúde e exposição às queimadas. Para cada incremento de $\mathrm{PM}_{10}$ de $50 \mu \mathrm{g} / \mathrm{m}^{3}$ a $150 \mu \mathrm{g} /$ $\mathrm{m}^{3}$ foram verificados aumentos de $12 \%$ nos atendimentos por doenças do trato respiratório superior, $37 \%$ por asma e $26 \%$ por rinite. Não foram encontradas associações para internações e para mortalidade.

Em análise específica para o período de incêndios na Malásia, Sastry ${ }^{71}$ investigou os efeitos das queimadas na mortalidade geral, excluindose aquelas por causas externas, respiratórias e cardiovasculares. Como modelagem estatística, os autores ajustaram uma regressão de Poisson via modelos lineares generalizados, examinando os efeitos do $\mathrm{PM}_{10}$ na mortalidade em diferentes grupos etários: < 1 ano, 1-64 anos, 65-74 anos, e 75 ou mais anos de idade. Foram encontrados resultados significativos para idosos em 4 e 5 dias após a exposição. Em análise semelhante, porém para dados de hospitalizações, também na Malásia, Mott et al..$^{70}$ investigaram os efeitos cardiorrespiratórios da exposição à fumaça. Análises de séries temporais por modelagem de HoltWinters indicaram que o total mensal de internações por causas respiratórias durante a época de queimadas superou, de maneira significativa, estimativas previstas com base em dados históricos, especificamente para internações por doença pulmonar obstrutiva crônica (DPOC) e asma. Além disso, técnicas de análise de sobrevi- da indicaram que pacientes com mais de 65 anos, admitidos anteriormente, devido outras causas, doenças cardiorrespiratórias, respiratórias ou DPOC, apresentaram maiores probabilidades de serem re-hospitalizadas durante o período de 1997 do que durante os anos de 1995 e 1996. Comunidades expostas à fumaça dos incêndios florestais apresentaram um incremento das hospitalizações por doenças cardiorrespiratórias.

\section{Austrália}

As queimadas não são um fenômeno recente na Austrália, embora a presença cada vez maior de comunidades residindo em áreas próximas de florestas tenha aumentado o acúmulo de biomassa para incêndios e o risco de danos maiores para a saúde humana ${ }^{73}$. Como consequência de longos períodos de seca e alterações de padrões climáticos, as queimadas têm-se tornado mais frequentes, começando em épocas anteriores às de costume e sendo mais duradouras ${ }^{74}$. Historicamente, naquele país, o período mais propício para queimadas tem sido entre os meses de dezembro e março. Entretanto, nos últimos anos, a estação de queimadas parece estar começando antes ${ }^{74,75}$.

A Austrália talvez seja o local, dentre os citados neste trabalho, que concentra a maior variedade de estudos sobre efeitos da poluição atmosférica na saúde humana. O principal objetivo desses estudos tem sido encontrar alguma associação entre a qualidade do ar e os desfechos de saúde.

Os efeitos à saúde que os investigadores escolheram estudar na Austrália têm variado de um estudo para outro. Alguns optaram por avaliar as mortalidades por todas as causas e as por doenças específicas como desfechos de saúde. Outros têm estudado internações por todas as causas e por doenças respiratórias, atendimentos em serviços de emergência para asma, função pulmonar entre outros ${ }^{22,76-79}$.

Os estudos sobre os efeitos da poluição por queimadas na Austrália têm sido desenvolvidos, principalmente, nos Estados de Melbourne, Sydney, Brisbane, Darwin e Victoria, pois são os locais que concentram a maior parte dos incêndios florestais daquele país ${ }^{42}$.

Durante o ano de 1994, incêndios florestais próximos a Sydney eleveram os níveis máximos de $\mathrm{PM}_{10}$ para valores de $250 \mu \mathrm{g} / \mathrm{m}^{3}$, aproximadamente, por um período de 7 dias $^{21}$. Dois estudos com dados de atendimentos de emergência por asma durante esses episódios de queimadas falharam em detectar quaisquer associações com a exposição à fumaça ${ }^{77,79}$. 
Smith et al. ${ }^{79}$ coletaram dados para dois períodos de 6 semanas cada: um para análise e o outro para controle. A análise conduzida adotou duas abordagens complementares. A primeira avaliou diferenças entre proporções de todos os atendimentos de emergência por asma nos dois períodos, não encontrando nenhuma diferença significativa entre ambos. Numa segunda etapa de análise foram ajustadas regressões de Poisson via modelos lineares generalizados, utilizando como desfechos o número diário de atendimentos de emergência por asma e por doenças respiratórias obstrutivas. Como variáveis de controle foram consideradas temperatura, umidade, pressão barométrica, velocidade do vento, precipitação, $\mathrm{PM}_{10}, \mathrm{O}_{3}$ e $\mathrm{NO}_{2}$. Nenhuma associação significativa entre os poluentes e os desfechos de saúde foi detectada. Embora, esta análise não tenha detectado nenhuma associação significativa com a poluição das queimadas, este estudo é extremamente limitado por utilizar apenas um período de controle. Além disso, as regressões de Poisson tinham um poder estatístico muito limitado, pois utilizaram apenas um mês para a análise de dados.

Numa análise mais recente deste mesmo episódio de poluição atmosférica, Jalaludin et al. ${ }^{77}$ relacionaram a exposição à fumaça com a capacidade pulmonar de crianças, não encontrando nenhuma associação significativa nem com $\mathrm{PM}_{10}$, nem com uma variável indicadora utilizada para representar o período de ocorrência dos incêndios florestais. Com base em dados diários para o período de $1^{\circ}$ de janeiro de 1994 a 31 de janeiro de 1994, os autores utilizaram técnicas de Equações de Estimação Generalizada, via modelos lineares generalizados. Não foram encontradas associações significativas nem para valores correntes e defasados em até quatro dias, nem para médias móveis de até ordem 5.

Em estudo realizado na cidade de Darwin, Johnston et al. ${ }^{80}$ analisaram a relação entre a concentração média diária de $\mathrm{PM}_{10}$ resultante da queima de vegetação e as internações hospitalares por asma. As internações ocorridas durante a estação seca do ano de 2000, entre abril e outubro, foram analisadas por meio de regressões de binomial negativa. Os autores justificam a utilização de uma variável binomial negativa, em detrimento ao uso de uma Poisson, afirmando que esta escolha é mais adequada para dados de contagem em que há sobredispersão. Nestes casos, o modelo de Poisson subestima a verdadeira variância, fornecendo resultados equivocados. Houve um aumento significativo nas internações por asma para cada aumento de $10 \mu \mathrm{g} / \mathrm{m}^{3}$ de $\mathrm{PM}_{10}$, sendo o efeito mais forte observado nos dias em que a concentração de $\mathrm{PM}_{10}$ superou 40 $\mu \mathrm{g} / \mathrm{m}^{3}$ em comparação com o período no qual os níveis de $\mathrm{PM}_{10}$ estavam inferiores a $10 \mu \mathrm{g} / \mathrm{m}^{3}$. Ao contrário dos estudos anteriores, este teve poder estatístico suficiente para detectar associações entre a exposição e o desfecho de saúde.

O impacto na saúde humana de incêndios florestais ocorridos em Brisbane foi analisado por meio de modelos lineares generalizados com regressão de binomial negativa no período de $1^{\circ}$ de julho de 1997 a 31 de dezembro de $2000^{81}$. Os autores optaram por trabalhar com a variável de exposição $\left(\mathrm{PM}_{10}\right)$ categorizada em três classes: baixa $(<$ 15), média (15-20) e alta (>20), ao invés de considerá-la com uma variável contínua. Foram encontradas associações em ambos os períodos: durante os incêndios florestais e no de ausência. As maiores associações foram estimadas para as categorias superiores de PM10 na época dos incêndios $(\mathrm{RR}=1,19, \mathrm{CI} 95 \%=1,09-1,30)$ e na ocasião de ausência destes ( $\mathrm{RR}=1,13$, CI95\% $=1,06-1,23)$.

Mais recentemente, com base numa série temporal de 1996 a 2005 de internações hospitalares por doenças cardiorrespiratórias da cidade de Darwin, Hanigan et al. ${ }^{76}$ investigaram a associação desse desfecho de saúde com a exposição ao $\mathrm{PM}_{10}$. Os autores utilizaram um modelo de Poisson via modelos lineares generalizados com sobredispersão e funções paramétricas suavizadoras para o tempo calendário e condições meteorológicas. Foram encontradas associações positivas e negativas, porém com grandes intervalos de confiança. Um aumento de $10 \mu \mathrm{g} / \mathrm{m}^{3}$ no mesmo dia de $\mathrm{PM}_{10}$ estava associado com um crescimento de 4,8\% (CI95\% $=-1,04-11,01 \%)$ nas internações por causas respiratórias.

\section{Discussão}

Esta breve revisão apresentou um panorama dos estudos de séries temporais de efeitos da poluição atmosférica por queimadas na saúde humana nas regiões que concentram o maior número de queimadas do planeta.

Enquanto estudos convencionais de séries temporais de efeitos da poluição atmosférica em áreas urbanas encontram constantemente resultados agudos estatisticamente significativos e positivos com os diferentes desfechos de saúde avaliados, nem todas as pesquisas realizadas para analisar as consequências de queimadas apontam impactos significativos na saúde pública. 
A primeira potencial explicação para as inconsistências nos resultados está na escolha do modelo estatístico utilizado ${ }^{82}$. Estudos realizados para investigar efeitos de curto prazo da poluição atmosférica urbana, geralmente, utilizam séries temporais com um período longo de observação. Uma série temporal longa o suficiente possibilitaria a detecção de pequenos efeitos de curto prazo na saúde pública devido à exposição à poluição atmosférica. Episódios de queimadas costumam ter curta duração e isso dificulta a análise estatística por séries temporais, tendo em vista o pequeno número de observações disponíveis.

Além disso, não há um único modelo estatístico que seja adequado para estudar os efeitos das queimadas ou da poluição atmosférica urbana na saúde humana. Com base na literatura consultada, observamos que são feitas as mesmas escolhas de modelagem de séries temporais, independente da natureza da exposição à poluição. Os estudos incluem riscos relativos para a exposição às queimadas no dia corrente ou em até cinco dias, em média, anteriores ao desfecho de saúde analisado, o que é conhecido na literatura como efeito defasado. Nenhum estudo consultado verificou o potencial efeito prolongado da exposição às queimadas para períodos maiores que uma semana ou um mês.

A pesquisa dos efeitos da poluição atmosférica por queimadas tende a focar, também, nos desfechos de mortalidade e hospitalizações. Tais efeitos representariam as consequências mais se- veras da exposição à fumaça das queimadas. Outros estudos apontam como explicações para as lacunas de conhecimento na pesquisa de efeitos da poluição atmosférica por queimadas, diferenças na composição química entre a destas e a de origem urbana ${ }^{83}$ e uma potencial não linearidade na função dose-resposta do $\mathrm{PM}^{84}$.

Vedal e Dutton ${ }^{83}$ argumentam que a combustão por combustíveis fósseis, geralmente, contém PM com metais e que estudos toxicológicos têm mostrado que tais partículas são mais danosas à saúde humana que aquelas sem metais. Os autores sugerem ainda que o PM de queimadas, que não possuem essa característica, poderia ser menos tóxico que aquele emitido em centros urbanos.

Para Martin et al. ${ }^{84}$, as queimadas emitem poluentes por um período curto de tempo, porém em elevados níveis de exposição. Os autores informam ainda que em níveis moderados a função dose-resposta do PM é praticamente linear. Entretanto, em níveis elevados, sua forma funcional ainda é incerta. E, nesse caso, utilizar a função dose-resposta obtida de estudos de poluição em centros urbanos poderia trazer viés aos resultados de impactos da poluição atmosférica por queimadas na saúde humana.

Reconhecendo as limitações do conhecimento atual e a necessidade de informações adicionais, análises futuras de efeitos da poluição de queimadas na saúde deveriam focar nessas importantes questões de pesquisa supracitadas.

\section{Colaboradores}

CN Carmo e SS Hacon participaram igualmente em todas as fases de elaboração do artigo. 


\section{Referências}

1. Gonçalves KS, Castro HA, Hacon SS. As queimadas na região amazônica e o adoecimento respiratório. Cien Saude Colet 2012; 17(6):1523-1532.

2. Tacconi L, Moore PF, Kaimowitz D. Fires in tropical forests: what is really the problem?: lessons from Indonesia. Mitigation and Adaptation Strategies for Global Change 2007; 12(1):55-66.

3. Cochrane MA, Schulze MD. Fire as a recurrent event in tropical forests of the eastern amazon: effects on forest structure, biomass, and species composition. Biotropica 1999; 31(1):2-16.

4. Peres F. Saúde, trabalho e ambiente no meio rural brasileiro. Cien Saude Colet 2009; 14(6):1995-2004.

5. Emmanuel SC. Impact to lung health of haze from forest fires: the Singapore experience. Respirology 2000; 5(2):175-182.

6. Varma A. The economics of slash and burn: a case study of the 1997-1998 Indonesian forest ûres. Ecol econ 2003; 46(1):159-171.

7. Keim ME. Building human resilience: the role of public health preparedness and response as an adaptation to climate change. Am j prev med 2008; 35(5): 508-516.

8. Wu J, Winer AM, Delfino RJ. Exposure assessment of particulate matter air pollution before, during, and after the 2003 Southern California wildfires. Atmos environ 2006; 40(18):3333-3348.

9. Cameron PA, Mitra B, Fitzgerald M, Scheinkestel CD, Stripp A, Batey C, Niggemeyer L, Truesdale M, Holman P, Mehra R, Wasiak J, Cleland H. Black saturday: the immediate impact of the February 2009 bushfires in Victoria, Australia. Med J Aust 2009; 191(1):11-16.

10. Fearnside PM, Graça PMLA. BR-319: Brazil's Manaus-Porto Velho Highway and the potential impact of linking the arc of deforestation to central Amazonia. Environ manage 2006; 38(5):705-716.

11. Laurance WF. A crisis in the making: responses of Amazonian forests to land use and climate change. TREE 1998; 13(10):411-415.

12. Soares RV. Perfil dos incêndios florestais no Brasil de 1984 a 1987. Floresta 1988; 18(12):94-121.

13. Soares RV, Santos JF, Batista AC. Some details of Forest fire statistics in Brazil. Floresta 2009; 39(3): 691-697.

14. Pope CA. Epidemiology of fine particulate air pollution and human health: biologic mechanisms and who's at risk? Environ health perspect 2000; 108(Supl. 4):713-723.

15. Logan WPD. Mortality in the London fog incident. Lancet 1953; 1:336-338.

16. World Health Organization (WHO). Air quality guidelines: global update 2005. Geneva: World Health Organization; 2006. p 1-22.

17. Dockery DW, Pope CA, Xu X, Spengler JD, Ware JH, Fay ME, Ferris Jr. BG, Speizer FE. An association between air pollution and mortality in six U.S. cities. N Engl J Med 1993; 329(24):1753-1759.

18. Ren C, Tong S. Health effects of ambient air pollution - recent research development and contemporary methodological challenges. Environ health 2008; 7:56.
19. Zmirou D, Schwartz J, Saez M, Zanobetti A, Wojtyniak B, Touloumi G, Spix C, Ponce de León A, Le Moullec Y, Bacharova L, Schouten J, Pönkä A, Katsouyanni K. Time series analysis of air pollution and cause-specific mortality. Epidemiology 1998; 9(5):495-503.

20. Artaxo P, Gerab F, Yamasoe MA, Martins J. Fine mode aerosol composition at three long-term atmospheric monitoring sites in the Amazon Basin. J Geophys Res 1994; 99(11):22857-22868.

21. Naher LP, Brauer M, Lipsett M, Zelikoff JT, Simpson CD, Koenig JQ, Smith KR. Woodsmoke health effects: a review. Inhal Toxicol 2007; 19(1):67-106.

22. Morgan G, Sheppeard V, Khalaj B, Ayyar A, Lincoln D, Jalaludin B, Beard J, Corbett S, Lumley T. Effects of bushfire smoke on daily mortality and hospital admissions in Sydney, Australia. Epidemiology 2010; 21(1):47-55.

23. Dominici F, Sheppard L, Clyde M. Health effects of air pollution: a statistical review. Int stat rev 2003; 71(2):243-276.

24. Goh KT, Schwela D, Goldammer JG, Simpson O. Health guidelines for vegetation fire events. Geneva: World Health Organization; 1999.

25. Boman C, Forsberg B, Sandstrom T. Shedding new light on wood smoke: a risk factor for respiratory health. Eur respir j 2006; 27(3):446-447.

26. Ezzati M, Kammen DM. The health impacts of exposure to indoor air pollution from solid fuels in developing countries: knowledge, gaps and data needs. Environ health perspect 2002; 110(11):10571068.

27. Larson T, Koenig J. Wood smoke: emissions and noncancer respiratory effects. Annu Rev Public Health 1994; 15:133-156.

28. Bell T, Adams M. Smoke from wildfires and prescribed burning in Australia: effects on human health and ecosystems. In: Bytnerowicz A, Arbaugh MJ, Riebau AR, Andersen C, editors. Developments in environmental sciences. [S.1.]: Elsevier; 2008. v. 8: wildland fires and air pollution. p. 289-316.

29. Carmo CN, Hacon S, Longo KM, Freitas S, Ignotti E, Ponce de Leon A, Artaxo P. Associação entre material particulado de queimadas e doenças respiratórias na região sul da Amazônia brasileira. Rev panam salud publica 2010; 27(1):10-16.

30. Artaxo P, Gatti LV, Leal AMC, Longo KM, Freitas SR, Lara LL, Pauliquevis TM, Procópio AS, Rizzo LV. Química atmosférica na Amazônia: a floresta e as emissões de queimadas controlando a composição da atmosfera amazônica. Acta Amazônica 2005; 35(2):185-196.

31. Nepstad D, Carvalho G, Barros AC, Alencar A, Capobianco JP, Bishop J, Moutinho P, Lefebvre P. Silva Jr. UL, Prins E. Road Paving, fire regime feedbacks, and the future of Amazon forests. Forests Ecology and Management 2001; 154(3):395-407.

32. Arbex MA, Cançado JED, Pereira LAA, Braga ALF, Saldiva PHN. Queima de biomassa e efeitos sobre a saúde. J bras pneumol 2004; 30(2):158-175. 
33. Malilay JA. Review of factors affecting the human health impacts of air pollutants from forest fires. In: World Health Organization (WHO). Background papers of Health guidelines for vegetation fire events. Genebra: WHO; 1999.

34. Cormier AS, Lomnicki S, Backes W, Dellinger B. Origin and health impacts of emissions of toxic byproducts and fine particles from combustion and thermal treatment of hazardous wastes and materials. Environ health perspect 2006; 114(6):810-817.

35. Phuleria HC, Fine PM. Air quality impacts of the October 2003 Southern California wildfires. J geophys res 2005; 110(D7):D07S20.

36. Environmental Protection Agency (EPA) (US). 40 CFR Part 50: revisions to the national ambient air quality standards for particulate matter: final rules. US: EPA; 1987. (The Federal Register 52, 24634-69.

37. Environmental Protection Agency (EPA) (US), 2006. 40 CFR Part 50: national ambient air quality standards for particulate matter: final rules. US: EPA; 2006. (The Federal Register 71, 61144-233).

38. Brauer M. Health impacts of biomass air pollution. In: Goh KT, Schwela D, Goldammer JG, Simpson $\mathrm{O}$, organizadores. Health guidelines for vegetation ûre events: background papers. Lima: World Health Organisation; 1999. p. 186-257.

39. Brasil. Ministério do Meio Ambiente. Conselho Nacional de Meio Ambiente. Resolução Conama $n^{\circ} 003$, de 28 de junho de 1990. Diário Oficial da União 1990; 22 ago.

40. Ignotti E, Hacon SS, Junger WL, Mourão D, Longo K, Freitas S, Artaxo P, Leon ACMP. Air pollution and hospital admissions for respiratory diseases in the subequatorial Amazon: a time series approach. Cad saude publica 2010; 26(4):747-761.

41. Mascarenhas MDM, Vieira LC, Lanzieri TM, Leal APPR, Duarte AF, Hatch DL. Poluição atmosférica devido à queima de biomassa florestal e atendimentos de emergência por doença respiratória em Rio Branco, Brasil - setembro, 2005. J bras pneumol 2008; 34(1):42-46.

42. Dennekamp M, Abramson MJ. The effects of bushfire smoke on respiratory health. Respirology 2011; 16(2):198-209.

43. Heil A, Goldammer JG. Smoke-haze pollution: a review of the 1997 episode in Southeast Asia. Regional Environmental Change 2001; 2(1):24-37.

44. Radojevic M, Hassan H. Air quality in Brunei Darussalam during the 1998 haze episode. Atmos environ 1999; 33(22):3651-3658.

45. Cope ME, Hess GD, Lee S, Tory K, Azzi M, Carras J, Lilley W, Manins PC, Nelson P, Nge L, Puri K, Wong N, Walsh S, Young M. The Australian Air Quality Forecasting System. Part I: Project Description and Early Outcomes. Journal of Applied Meteorology 2004; 43(5):649-662.

46. Aires CB, Kirchhoff VWJH. Transporte de monóxido de carbono gerado em queimadas para regiões onde não se queima. Rev Bras Geof 2001; 19(1):6174 .
47. Brasil. Ministério da Ciência e Tecnologia e Inovação, Ministério do Meio Ambiente. Monitoramento de incêndios e queimadas por satellite em tempo quasereal. [página na Internet]. [acessado 2013 set 9]. Disponível em: http://www.cptec.inpe.br/queimadas

48. Longo KM, Freitas SR, Setzer A, Prins E, Artaxo P, Andreae $\mathrm{M}$. The coupled aerosol and tracer transport model to the brazilian developments on the regional atmospheric modeling system (CATTBRAMS) - Part 2: model sensitivity to the biomass burning inventories. Atmospheric Chemistry and Physics 2007; 7:8571-8595.

49. Bell ML, Samet JM, Dominici F. Time-series studies of particulate matter. Annu Rev Public Health 2004; 25:247-280.

50. Dominici F, Mcdermott A, Zeger SL, Samet J. On the use of generalized additive models in time-series studies of air pollution and health. Am J Epidemiol 2002; 156(3):193-203.

51. Greenbaum DS, Bachmann JD, Krewski D, Samet JM, White R, Wyzga R. Particulate air pollution standards and morbidity and mortality: case study. Am J Epidemiol 2001; 154(12 Supl.):78-90.

52. Pan American Health Organization (PAHO). An assessment of Health effects of Ambient Air Pollution in Latin America and the Caribbean. Washington: PAHO; 2005.

53. Schwartz J, Marcus A. Mortality and air pollution in London: a time series analysis. Am J Epidemiol 1990; 131(1):185-194.

54. Schwartz J, Spix C, Touloumi G, Bacharova L, Barumamdzadeh T, Tertre A, Piekarksi T, Leon AP, Ponka A, Rossi G, Saez M, Schouten JP. Methodological issues in studies of air pollution and daily counts of deaths or hospital admissions. J epidemiol community health 1996; 50(Supl. 1):S3-S11.

55. Stieb DM, Judek S, Burnett RT. Meta-Analysis of time-series studies of air pollution and mortality: effects of gases and particles and the influence of cause of death, age and season. Journal of the Air \& Waste Management Association 2004; 52(4):470-484.

56. Dobson AJ. An introduction to generalized linear models. $2^{\text {nd }}$ Edition. New York: Chapman \& Hall; 2002.

57. He S, Mazumdar S, Arena VC. A comparative study of the use of GAM and GLM in air pollution research. Environmetrics 2006; 17(1):81-93.

58. Brown IF, Schroeder W, Setzer A, Maldonado MR, Pantoja N, Duarte A, Marengo J. Monitoring fires in southwestern Amazonia rain forests. EOS, Transactions 2006; 87(26):253-264.

59. Ignotti E, Hacon SS, Silva AMC, Junger WL, Castro H. Efeitos das queimadas na Amazônia: método de seleção dos municípios segundo indicadores de saúde. Rev bras epidemiol 2007; 10(4):453-464.

60. Fried JS, Torn MS, Mills E. The impact of climate change on wildfire severity: a regional forecast for northern California. Clim change 2004; 64(1-2):169191.

61. Safford HD. Man and ûre in Southern California: doing the math. Fremontia 2007; 35(4):25-29. 
62. Bytnerowicz A, Cayan D, Riggan P, Schilling S, Dawson P, Tyree M, Wolden L, Tissell R, Preisler H. Analysis of the effects of combustion emissions and Santa Ana winds on ambient ozone during the October 2007 southern California wildûres. Atmos environ 2010; 44:678-687.

63. Westerling AL, Hidalgo HG, Cayan DR, Swetnam TW. Increases in Western US forest wildfire associated with warming and advances in the timing of spring. Science 2006; 313:940-943.

64. Duclos P, Sanderson LM, Lipsett M. The 1987 forest fire disaster in California: assessment of emergency room visits. Arch Environ Health 1990; 45(1):53-58.

65. Kunzli N, Avol E, Wu J, Gauderman WJ, Rappaport E, Millstein J, Bennion J, McConnell R, Gillilan FD, Berhane K, Lurmann F, Winer A, Peters J. Health effects of the 2003 Southern California wildfires on children. Am J Respir Crit Care Med 2006; 174(11):1221-1228.

66. Mott JA, Meyer P, Mannino D, Redd SC. Wildland forest fire smoke: health effects and intervention evaluation, Hoopa, California, 1999. West J Med 2002; 176(3):157-162.

67. Jacobs J, Kreutzer R, Smith D. Rice burning and asthma hospitalizations, Butte County, California, 1983-1992. Environ health perspect 1997; 105(9):980985.

68. Viswanathan S, Eris L, Diunugala N, Johnson J, McClean C. An analysis of effects of San Diego wildfire on ambient air quality. Journal of Air Waste Management Association 2006; 56(1):56-67.

69. Field RD, Shen SSP. Predictability of carbon emissions from biomass burning in Indonesia from 1997 to 2006. J geophys res 2008; 113(G4):G04024.

70. Mott JA, Mannino DM, Alverson CJ, Kiyu A, Hashim J, Lee T, Falter K, Redd SC. Cardiorespiratory hospitalizations associated with smoke exposure during the 1997, Southeast Asian forest fires. Int $j$ hyg environ health 2005; 208(1-2):75-85.

71. Sastry N. Forest Fires, air pollution, and mortality in Southeast Asia. Demography 2002; 39(1):1-23.

72. Chew FT, Goh DYT, Ooi BC, Saharom R, Hui JKS, Lee BW. Association of ambient air-pollution levels with acute asthma exacerbation among children in Singapore. Allergy 1999; 54(4):320-329.

73. Sim M. Bushfires: are we doing enough to reduce the human impact? Occup environ med 2002; 59(4):215-216.

74. Tham R, Erbas B, Akram M, Dennekamp M, Abramson $\mathrm{M}$. The impact of smoke on respiratory hospital outcomes during the 2002-2003 bushure season, Victoria, Australia. Respirology 2009; 14(1):69-75.
75. Taylor J, Webb R. Meteorological aspects of the January 2003 south-eastern Australia bushfire outbreak. Australian Forestry 2005; 68(2):94-103.

76. Hanigan IC, Johnston FH, Morgan GG. Vegetation fire smoke, indigenous status and cardio-respiratory hospital admissions in Darwin, Australia, 19962005: a time-series study. Environ health 2008; 7:42.

77. Jalaludin B, Smith M, O'Toole B, Leeder S. Acute effects of bushûres on peak expiratory ûow rates in children with wheeze: a time series analysis. Aust N $Z j$ public health 2000; 24(2):174-177.

78. Johnston FH, Bailie RS, Pilotto LS, Hanigan IC. Ambient biomass smoke and cardio-respiratory hospital admissions in Darwin, Australia. BMC Public Health 2007; 7:240.

79. Smith MA, Jalaludin B, Byles JE, Lim L, Leeder SR. Asthma Presentations to Emergency Departments in Western Sydney during the January 1994 Bushfires. Int $j$ epidemiol 1996; 25(6):1227-1236.

80. Johnston FH, Kavanagh AM, Bowman DMJS, Scott RK. Exposure to bushfire smoke and asthma: an ecological study. Medical Journal of Australia 2002; 176(11):535-538.

81. Chen L, Verral K, Tong S. Air particulate pollution due to bushfires and respiratory hospital admissions in Brisbane, Australia. Int $j$ environ health res 2006; 16(3):181-191.

82. Erbas B, Hyndman RJ. Sensitivity of the estimated air pollution-respiratory admissions relationship to statistical model choice. Int $j$ environ health res 2005; 15(6):437-448.

83. Vedal S, Dutton SJ. Wildûre air pollution and daily mortality in a large urban area. Environ res 2006; 102(1):29-35.

84. Martin WE, Brajer V, Zeller K. Valuing the health effects of a prescribed fire. In: Martin WE, Raish C, Kent B, organizadores. Wildfire risk: human perceptions and management implications. Washington, DC: RFF; 2007. p. 244-261.

Artigo apresentado em 26/06/2012

Aprovado em 27/07/2012

Versão final apresentada em 02/08/2012 